\title{
Respuestas del Estado peruano frente al crimen organizado en el siglo XXI
}

DOI: https://doi.org/10.18046/recs.i31.3710

\author{
Peruvian State's Responses to Organized Crime \\ in the 21st Century
}

\author{
Sofía Vizcarra ${ }^{* *}$ \\ Universidade do Vale do Rio dos Sinos (Porto Alegre, Brasil)
}

Diana Bonilla ${ }^{* * *}$

Pontificia Universidad Católica del Perú (Lima, Perú)

\section{Bertha Prado ${ }^{* * * *}$}

Pontificia Universidad Católica del Perú (Lima, Perú)

\footnotetext{
* El presente artículo es producto del trabajo colaborativo de las autoras como investigadoras durante el funcionamiento del Laboratorio de Criminología de la Pontificia Universidad Católica del Perú. No se recibió financiamiento específico para el desarrollo de este proyecto. Artículo de investigación recibido el 15.09.2O19 y aceptado el 24.02.2020.

** Profesora del Departamento de Relaciones Internacionales de la Universidade do Vale do Rio dos Sinos (Unisinos) (Brasil).Correo electrónico: svizcarracas@unisinos.br ORCID: https://orcid.org/oooo-ooo1-7321-0074

*** Magíster y licenciada en Sociología de la Pontificia Universidad Católica del Perú (Perú). Investigadora adjunta del Grupo Interdisciplinario de Criminología de la Pontificia Universidad Católica del Perú (Perú). Correo electrónico: diana. bonilla@pucp.edu.pe ORCID: https://orcid.org/oooo-ooo1-6950-6817

**** Investigadora predoctoral en el programa de Doctorado en Ciencias Jurídicas y Sociales de la Universidad de Málaga (España). Miembro del grupo de investigación de Derecho Penal y Criminología de la Pontificia Universidad Católica del Perú (Perú). Correo electrónico: bprado@pucp.edu.pe ORCID: https://orcid.org/oooo-ooo1-6970-728o
} 


\section{Cómo citar/How to cite}

Vizcarra, Sofía; Bonilla, Diana; Prado, Bertha (2020). Respuestas del Estado peruano frente al crimen organizado en el siglo XXI. Revista CS, 31, 109 - 138.

https://doi.org/10.18046/recs.i31.3710 


\section{Resumen}

La lucha contra el crimen organizado se ha convertido en uno de los ejes prioritarios de la política criminal del Estado peruano. Como consecuencia de ello, el Gobierno de este país ha aprobado una serie de medidas orientadas a controlar dicho fenómeno. El objetivo principal de este artículo es describir, desde un enfoque teórico y empírico, el panorama general de las manifestaciones del crimen organizado en el Perú y las respuestas del Estado para controlarlo. En ese contexto, se plantea identificar la existencia, o no, de elementos suficientes para configurar una política general eficiente contra el crimen organizado. Para ello, se realizó una revisión normativa y de archivo, que permite valorar críticamente los avances y desafíos de las respuestas del Estado para afrontar y sostener una política más eficiente de control y lucha contra las organizaciones criminales en el Perú.

PALABRAS CLAVE:

crimen organizado, política criminal, organización criminal, Perú

The fight against organized crime has become one of the priorities of the Peruvian State's criminal policy. As a result, the Peruvian government has approved different measures to control this phenomenon. The aim of this paper is to describe, from a theoretical and empirical approach, the overall picture of the manifestations of organized crime in Peru and the State's responses to control it. In this context, it is proposed to identify the existence, or not, of sufficient elements to configure an efficient general policy against organized crime. In order to do so, a legislation and records review was carried out, which allows to critically assess the progress and challenges of the State's responses to face and sustain a more efficient policy of control and fight against criminal organizations in Peru.

\section{KEYWORDS:}

Organized Crime, Criminal Policy, Criminal Organization, Peru 



\section{Introducción}

La adopción de la Convención de las Naciones Unidas contra la Delincuencia Transnacional (Convención de Palermo) marcó la formalización de la definición de un nuevo problema público de escala global: el crimen organizado transnacional. Así, a pesar de que la transnacionalización de fenómenos criminales no es reciente (Andreas, 2011), sí lo es su priorización como una de las principales amenazas globales del siglo XXI. Esta agenda global llevó a que los países signatarios de la Convención de Palermo adoptaran legislaciones específicas en la materia y priorizaran este combate en sus fronteras.

En el Perú, la legislación sobre crimen organizado (Ley 30 077) fue adoptada en 2013. Esta ley creó una categoría procesal específica relacionada a los delitos cometidos por organizaciones criminales con determinadas características, y definió técnicas especiales de investigación adaptadas a la complejidad de estos delitos. La puesta en operación de esta ley fue progresiva, pero su uso se ha intensificado a partir de 2016. En dicho año, el Gobierno peruano entrante priorizó la lucha contra el crimen organizado a través de la implementación de megaoperativos policiales destinados a la captura y desarticulación de organizaciones criminales. Esta estrategia marcó un cambio en el lugar que el crimen organizado ocupaba en la política de seguridad, y tuvo resultados operativos y mediáticos de impacto, principalmente con relación a la contención del uso de la fuerza para el combate contra el crimen organizado y al uso intensivo de estrategias de inteligencia policial. Sin embargo, los cambios en las gestiones presidenciales y ministeriales llevaron a que dicha prioridad se perdiera, aunque el tema continuó en la agenda mediática a través del uso de la categoría crimen organizado en los más importantes casos de corrupción a nivel nacional ${ }^{1}$, y aquellos relacionados con el caso Lava Jato ${ }^{2}$, a nivel regional.

La coyuntura política en el Perú, a partir de 2018, año marcado por el destape de dichos casos, llevó a que diferentes operadores relacionados con el combate contra el crimen organizado fueran cuestionados y fiscalizados desde diferentes tribunas mediáticas y políticas. Más allá de la coyuntura, estos cuestionamientos

1. En el Perú, la categoría de organización criminal también ha sido empleada para las investigaciones de casos como el del partido político Fuerza Popular, acusado de lavado de activos, y el caso de autoridades judiciales conocido a partir de los llamados CNM Audios, presuntamente involucradas en tráfico de influencias y sobornos, entre otros delitos de corrupción en los más altos niveles del poder judicial.

2. Durante los últimos años, se han descubierto importantes casos de corrupción en la región, asociados a la empresa de construcción Odebrecht. Las investigaciones al respecto iniciaron en Brasil y se denominaron Operación Lava Jato. En el caso peruano, estos casos involucran a los últimos cuatro presidentes de la República (Alejandro Toledo, Ollanta Humala, Alan García y Pedro Pablo Kuczynski), que habrían recibido pagos ilegales por parte de dicha empresa a cambio de facilitar o conceder licitaciones de obras públicas. 
evidenciaron desconocimiento en la materia y poca claridad sobre cómo el Estado estaba abordando el combate contra este fenómeno. Por ello, el presente artículo busca presentar un panorama general de las respuestas del Estado peruano al crimen organizado en el siglo XXI. Específicamente, las indagaciones se orientan a verificar si existen indicios suficientes para identificar una política general del Estado peruano contra el crimen organizado (formalizada o no como tal) con un enfoque comprehensivo y coherente. Una política de este tipo implicaría un enfoque teórico y analítico común sobre el combate a diferentes delitos, pero que podría expresarse en más de un documento de política que se adapte a las características específicas de las manifestaciones de crimen organizado en el país.

Así, a partir de una revisión de documentos normativos, memorias de gestión, archivos de prensa correspondientes al período 2013-2019 (a partir de la entrada en vigor de la ley contra el Crimen Organizado en el Perú), así como entrevistas informales con algunos operadores del sistema de seguridad y justicia, este artículo realiza un balance sobre los avances y desafíos de las respuestas del Estado peruano para combatir el crimen organizado. Para ello, la primera sección identifica los principales enfoques teóricos a partir de los cuales se comprende este fenómeno y se estructuran las respuestas estatales para su combate. La segunda describe brevemente las principales características de las manifestaciones de crimen organizado más relevantes en el país. La tercera hace un balance de las normas, planes y estrategias existentes en la materia, identificando tanto fortalezas como debilidades. Finalmente, la cuarta sección aborda tres de los principales desafíos del Estado peruano para la consolidación de una política integral contra el crimen organizado.

\section{Consideraciones teóricas para comprender los enfoques de combate al crimen organizado}

El crimen organizado es un concepto polisémico. Existen al menos dos ámbitos para el uso del concepto: el académico, donde es una categoría analítica de fenómenos sociales; y el de la política pública de seguridad, donde funciona como definición de un problema público y base para definir políticas, estrategias e instrumentos de seguridad pública que permitan contrarrestarlo. En este último ámbito, además, la categorización de un grupo de personas como organización criminal permite a los operadores del sistema penal utilizar técnicas especiales de investigación para sus indagaciones (levantamiento del secreto de las comunicaciones, seguimientos, etc.).

Desde la perspectiva académica, no hay consenso acerca de la definición de crimen organizado. Así, existen más de doscientas definiciones de él (Von Lampe, 
s.f.). Además, el concepto varía y prioriza diferentes elementos en función del contexto sociohistórico desde el cual se discute (Varese, 2017). Sin embargo, la mayoría de las definiciones se articulan en torno a dos dimensiones del concepto: por una parte, la noción de estructura de una organización criminal; y, por otra, la noción de actividad criminal de las organizaciones.

La criminología ha profundizado en el estudio de estas dos dimensiones (Paoli, 2014), pues ambas son útiles tanto para comprender el fenómeno delictivo como para pensar las respuestas de política pública pertinentes. No obstante, el foco en la estructura de la organización es un "elemento central en la configuración de la imputación concreta apropiada" (Sala Penal Nacional, 2017: 2-3) del crimen organizado. Esta perspectiva se centra en cuestiones relacionadas con los autores de delitos y las formas de organización que emplean para repartir tareas y asignar funciones. Por ello, una noción importante para estudiar el crimen organizado es la de crimen asociativo, la cual parte de una premisa importante: las estructuras criminales tienen un componente social inminente, ya que los actores criminales interactúan necesariamente en un contexto y espacio determinados (Van de Bunt; Siegel; Zaitch, 2014; Von Lampe, 2016). Asimismo, muchas de estas estructuras se configuran de manera similar, ya que funcionan a partir de mecanismos parecidos; por ejemplo, sostener lazos de confianza y apoyo mutuo.

Las organizaciones de tipo mafia (Paoli, 2014; 2016) han recibido amplia atención en la literatura internacional. Esta estructura jerárquica, con una cadena de mando definida y que usa medios armados o violentos para garantizar el respeto de las decisiones, se ha identificado en diversos contextos, como Italia (Gambetta, 1993; Paoli, 2003), Rusia (Volkov, 2014), China (Chin, 2014; Chu, 2005) o Japón (Hill, 2003). Aunque existen especificidades locales y escalas diferentes, este tipo de estructuras, en general, están conectadas con el poder político local y ejercen un cierto control territorial. La asociación del crimen organizado con estructuras tipo mafia fue una tendencia en la academia durante las décadas de 1960 y 1980. Luego, el desarrollo de la investigación en diferentes países llevó a los operadores y académicos a abandonar el modelo Cosa Nostra como el único paradigma organizacional, aunque durante los años 1990 y 200o, el elemento jerárquico siguió siendo central para las principales definiciones de crimen organizado (Varese, 2017: 31).

Sin embargo, como señala Paoli (2016), las organizaciones de tipo mafia están lejos de dominar algunos tipos de crimen organizado, como los mercados ilegales. Por el contrario, como señala Reuter (1983), la mayoría de los intercambios ilegales en los países occidentales se llevan a cabo por numerosas empresas relativamente pequeñas y a menudo efímeras, a las que denomina crimen desorganizado. Dichas organizaciones pueden tener patrones de organización no jerárquicos, estructuras 
flexibles como la configuración en red y tener roles específicos para el tipo de actividad ilegal realizada. Ello implica que los operadores de seguridad y justicia deban identificar y comprender el espectro completo de patrones de organización criminal, que van desde organizaciones de tipo mafia hasta el crimen desorganizado.

En ese sentido, organizaciones internacionales han intentado contribuir a construir tipos ideales de estructuras, para facilitar la aplicación del concepto de crimen organizado. Como afirma la Oficina de Naciones Unidas para la Droga y el Delito (UNODC, 2002), estos tipos ideales son:1) las jerarquías estándar, con un solo líder, jerarquías y roles definidos, estrictos regímenes disciplinarios y una fuerte lealtad hacia el líder; 2) las jerarquías regionales, con un liderazgo centralizado, pero autonomías locales; 3 ) los clúster, asociaciones de diversos grupos criminales; 4) el grupo central; y 5) la red criminal, definida por las actividades de ciertos actores clave que forman alianzas para fines específicos.

A partir de la problematización de estos tipos ideales, algunos autores sostienen que es posible identificar, más bien, una relación de tránsito entre estos tipos de estructuras en los últimos años. Es decir, que muchas organizaciones pueden transformar sus patrones de organización desde jerarquías estándar hasta redes más laxas (Holmes, 2009; Morselli, 2009). Otros autores, en los esfuerzos por sistematizar la producción acerca de patrones de organización criminal y nuevos modelos de estructuras, consideran que en la realidad los tipos ideales se interrelacionan y transforman constantemente, y que sus límites son más bien difusos (Le, 2012). Entonces, los múltiples esfuerzos por definir al crimen organizado, así como sus principales formas sirven más para diferenciar con claridad qué es crimen organizado y qué no, en los casos más cercanos a los tipos ideales; pero en otros puede ser más útil identificar el grado o ubicación del caso en un continuo de criminalidad organizada (Hagan, 1983). Así, se puede tomar la noción de crimen organizado, en general, como un tipo ideal, que tiene mayor utilidad cuando se trata como una construcción analítica con base en la cual nos aproximamos a fenómenos empíricos de criminalidad organizada.

Desde la perspectiva de política pública, la noción de crimen organizado corresponde a la definición de un problema público en un determinado contexto que orienta las respuestas del Estado frente a él. En ese sentido, en el Perú, el enfoque centrado en las estructuras y los patrones de organización es predominante en la normativa vigente y, por lo tanto, se traduce en la política pública existente. Así, la definición jurídico-penal operante, que se desprende del Artículo 317 del Código Penal, y la Ley 30077 (Ley contra el Crimen Organizado), plantea los criterios a partir de los cuales se define una organización criminal. Estos son que la agrupación sea de tres o más personas, que exista una repartición de tareas o funciones, que la organización 
tenga cierta estabilidad y permanencia en el tiempo, y que se dedique a la comisión de uno o más delitos graves señalados en dicha ley.

Reconociendo las dificultades de aplicación de estas normas para la caracterización de una estructura criminal, la Sala Penal Nacional del Poder Judicial del Perú señaló que existen diversos patrones de organización criminal y subrayó la importancia de identificar los roles de los miembros de una organización, sin que ello implique necesariamente la existencia de una jerarquía, como en los modelos tradicionales.

Sin embargo, un punto importante es que la normativa peruana diferencia organización criminal de banda criminal (Artículos 317-A y 317-B del Código Penal). Así, una organización tendría las características ya mencionadas, mientras que una banda criminal se definiría por ser una asociación de dos o más personas, que no necesariamente tiene una estructura definida, roles o permanencia en el tiempo, pero que tiene por objeto la comisión de delitos de manera concertada. En ese sentido, el problema público al que responden las políticas de crimen organizado corresponde a acciones de un tipo de criminalidad compleja, con una acción sostenible en el tiempo y asociada a uno o varios delitos considerados graves por la normativa vigente.

Por otro lado, el enfoque centrado en las actividades de una organización criminal comprende el análisis de las actividades legales e ilegales que contribuyen a la comisión de sus delitos. El propósito de este análisis, de estudiar el qué (Paoli, 2016), es mejorar la comprensión de las tareas, aportes y capacidades requeridas para la comisión de un determinado delito. Sin embargo, el alcance de las actividades delictivas que pueden ser investigadas por los operadores de justicia como crimen organizado son las definidas en la legislación de cada país.

Desde esta perspectiva, las organizaciones criminales se pueden clasificar de acuerdo con la actividad principal que les permite generar ingresos o beneficios económicos (Prado-Saldarriaga, 2019). En este sentido, para facilitar la comprensión de cómo generan ganancias las organizaciones criminales, es pertinente retomar la clasificación de Naylor (2003) sobre los delitos con fines de lucro que comprende: 1) delito predatorio, donde se extrae riqueza existente a través de actos ilegales (uso de la fuerza o engaño);2) mercados ilegales, donde se intercambian bienes ilegales en actos legales o ilegales (tráfico); y 3 ) delitos comerciales y corporativos, que se cometen en entidades legales mediante la transferencia de bienes legales a través de métodos ilegales (fraude). Si bien esta clasificación no solo aplica para el crimen organizado, es un ejercicio útil para comprender la diversidad de tareas y métodos existentes en organizaciones criminales.

Así, la complejidad del análisis del crimen organizado como fenómeno social y como problema público reside en el reconocimiento de su complejidad intrínseca, relacionada con las dos dimensiones que lo constituyen: las estructuras y las acti- 
vidades. En América Latina, y particularmente en el Perú, las diferentes políticas de seguridad, como veremos a continuación, tuvieron un énfasis marcado en el componente de actividades, creándose así políticas específicas por delitos considerados particularmente graves. Sin embargo, son el inicio de la comprensión del componente estructural y las nuevas dificultades planteadas por la adopción de la legislación sobre crimen organizado las que han comenzado a reconfigurar la política contra el crimen organizado en el país.

\section{El Perú y el problema del crimen organizado}

El concepto jurídico-penal de crimen organizado es de reciente aparición en el Perú. Sin embargo, las principales expresiones de este fenómeno en el país datan de décadas previas, y se pueden diferenciar en dos tipos, de acuerdo con un enfoque centrado en sus actividades: 1 ) aquellas relacionadas con mercados ilegales y 2) aquellas centradas en delitos predatorios.

Por un lado, las organizaciones relacionadas con los mercados ilegales pueden ser entendidas como empresas de carácter ilegal, cuya actividad principal se basa en la producción o venta ilegal de un servicio o bien a nivel local o trasnacional. Este es el caso de las organizaciones dedicas al narcotráfico, la trata de personas, la minería ilegal, entre otros, que pueden funcionar de manera jerárquica y en redes. En este ámbito, en el Perú resalta la situación del tráfico ilícito de drogas, especialmente de derivados cocaínicos.

Las primeras formas de crimen organizado de este tipo se pueden identificar, aproximadamente, a partir de finales de la década de 1970 y durante los años ochenta. Las organizaciones que tuvieron mayor visibilidad en este periodo eran firmas que estaban vinculadas principalmente al narcotráfico en los principales valles cocaleros del país: el Huallaga, el Monzón y el Vraem. En este período se encontraron estructuras jerárquicas más visibles, por lo que las cabezas de estas organizaciones narcotraficantes como Mosca Loca ${ }^{3}$ o Vaticano ${ }^{4}$, entre otros, adquieren notoriedad.

3. Guillermo Porto Cárdenas, alias Mosca Loca, fue un popular narcotraficante que desarrolló sus actividades entre las décadas de 1970 y 1980 en la selva peruana, y fue conocido por tener vínculos con importantes políticos de la época e incluso ofrecer pagar la deuda externa del país a cambio de impunidad para su actividad ilegal.

4. Demetrio Chávez Peñaherrera, alias Vaticano, fue otro narcotraficante peruano dedicado al tráfico ilícito de drogas en la zona del Alto Huallaga, entre las décadas de 1980 y 1990. Durante su juicio se hizo conocido que desarrollaba sus actividades gracias a un pago mensual que realizaba al principal asesor del entonces presidente Alberto Fujimori. 
Actualmente, el Perú es el segundo mayor productor de hoja de coca en el mundo, con una extensión de 49900 hectáreas de este cultivo a nivel nacional (UNODC, 2018), $90 \%$ del cual se destina al mercado ilegal, produciendo potencialmente alrededor de 400 toneladas de cocaína anuales. De dicha producción potencial, la División Antidrogas de la Policía Nacional del Perú (PNP, 2019) reporta un decomiso anual de 57,057 kg de drogas para el año 2018. Así, en la cadena de suministro de drogas a nivel global, el Perú se ubica, básicamente, en el eslabón de la producción, ya que el consumo nacional de cocaína tiene niveles muy bajos (Comisión Nacional para una Vida sin Drogas [Devida], 2014: 113).

Otro mercado ilegal importante en el Perú es el relacionado con la minería de oro, que genera altos niveles de rentabilidad. En este caso, los datos disponibles evidencian una diferencia importante entre la producción nacional y los volúmenes de exportación. De esta manera, desde inicios de la década del 2000, en un contexto económico favorable caracterizado por un aumento sostenido de los precios y la cotización del oro, la brecha entre estos dos indicadores se hace más notoria (Cuzcano, 2015). Ello se explica, en gran medida, por la incidencia de la actividad extractiva ilegal en el Perú, especialmente en zonas de selva como Madre de Dios y otras regiones de la costa norte.

Así, por ejemplo, en el período 2010-2013, la producción ilegal o informal representó aproximadamente la cuarta parte de la producción total de oro, y el valor total de dicha producción se estima en más de 15 ooo ooo ooo de dólares (Cuzcano, 2015). Mientras tanto, las fuerzas del orden reportan más de 400 operativos contra la minería ilegal a nivel nacional solo en 2018 (PNP, 2019). Otros mercados ilegales que operan en el país son la tala de madera, el contrabando y la trata de personas (Dammert; Bensús; Sarmiento; Prieto, 2017).

En el país, estos mercados ilegales se estructuran como cadenas de valor global (Gereffi; Humphrey; Kaplinsky; Sturgeon, 2001), siendo el Perú el punto cero de producción de estas commodities ilegales. Así, la estructura de las organizaciones en este tipo de mercados corresponde, en general, a pequeños núcleos familiares conectados mediante intermediarios (Vizcarra, 2018b) en una configuración de red acéfala, como ya se ha identificado en otros países productores de drogas cocaínicas, como Colombia (Kenney, 2007). Algunos de los núcleos que se dedican al segmento de exportación de estas cadenas de valor pueden llegar a desarrollar estructuras más jerárquicas, pero no es la regla dentro del mercado que opera en el país.

El segundo tipo de actividad a la cual se dedican las organizaciones criminales en el Perú es el crimen predatorio. Estas organizaciones no generan nuevos bienes o servicios para incrementar sus ganancias, por el contrario, sus actividades se caracterizan por la redistribución de riqueza de un actor a otro, a través de una transferencia 
involuntaria y bilateral de víctima a victimario, que genera pérdidas para la primera (Naylor, 2003). En el Perú, en la década de 1990, en medio de la crisis económica y política del país, continuaron operando las organizaciones criminales dedicadas al tráfico ilícito de drogas, pero empezaron a tener notoriedad este tipo de organizaciones criminales en las ciudades, dedicadas a delitos como el robo de bancos y secuestros a empresarios, especialmente en las ciudades de la costa centro y norte peruana. Estos delitos, en general, son cometidos mediante la amenaza o el uso de la violencia, y se les asocia al aumento de indicadores ligados a violencia interpersonal, como las tasas de homicidios por sicariato o la victimización con arma de fuego.

Si bien la medición del crimen organizado en este ámbito es compleja, algunos indicadores permiten aproximarnos al panorama general del accionar de estas organizaciones y su concentración territorial. Así, por ejemplo, según el Instituto Nacional de Estadística e Información (2018), las ciudades costeñas presentan mayor cantidad de víctimas por sicariato; mayores tasas de denuncias por extorsión, según el Ministerio Público (2018), y altas tasas de homicidio calificado, en comparación con el resto del país. Asimismo, de acuerdo con los informes estadísticos del Instituto Nacional Penitenciario (INPE, 2019), más de la mitad de los internos por el delito de extorsión en todo el país se encuentran en los establecimientos penitenciarios de Lima y Trujillo. Esto contribuye a una caracterización de algunas ciudades de la costa norte como violentas y peligrosas en la prensa y en el imaginario colectivo (Bonilla, 2019).

La estructura de las organizaciones que se dedican a este tipo de actividades ha variado con el transcurrir de los años. Así, a inicios de los años 200o, comenzó una cierta fragmentación de las grandes organizaciones criminales que operaban en la franja costera, las cuales se reestructuraron en organizaciones más pequeñas dedicadas a delitos menos visibles y con menor posibilidad de detección, como la extorsión. Finalmente, en años más recientes, se mantiene una lógica de fragmentación de organizaciones y reestructuración, especialmente en los establecimientos penitenciarios. Estos cobran un rol muy importante en las formas y patrones de organización de estos grupos, debido al desarrollo de nuevas tecnologías de comunicación y las fallas del Estado en el control de las comunicaciones en los penales, que facilitan la continuidad de las operaciones de criminales ya encarcelados, así como la reorganización de grupos desarticulados.

Así, las manifestaciones del crimen organizado en el Perú muestran la complejidad que existe detrás de esta etiqueta. Dadas las diferencias en las actividades, estructuras y modus operandi de las organizaciones criminales en el país, la respuesta del Estado ha sido, en general, fragmentada y específica para cada tipo de delito, como se señala a continuación. 


\section{¿Política o políticas contra el crimen organizado?}

En los últimos veinte años, el Estado peruano ha elaborado diferentes leyes, políticas, planes y estrategias orientados a combatir el crimen organizado. En el presente apartado se expondrá un breve recuento de los instrumentos de naturaleza política y legislativa aprobados, y se señalarán sus principales aportes o contradicciones de cara a aproximarse al análisis de la existencia y coherencia de una política contra el crimen organizado en el Perú.

\section{Lo que existe: políticas específicas para delitos específicos}

La lucha contra el crimen organizado en el Perú se caracteriza por contar con una gran cantidad de instrumentos normativos de naturaleza penal y procesal penal. Como se observa en el Cuadro 1, existe una amplia normativa dirigida a identificar aquellos delitos pasibles de ser calificados como crimen organizado y a establecer las pautas para su persecución, juzgamiento y sanción. También se distingue una normativa que se encarga de fortalecer los mecanismos penales para delitos concretos como el lavado de activos y la minería ilegal. Finalmente, se identifican normas orientadas a tipificar el delito de organización criminal, previsto en el Artículo 317 del Código Penal.

Por otro lado, y debido a la ambigüedad de la técnica legislativa de algunas de las normas penales mencionadas o a la complejidad de su aplicación, la Corte Suprema

\begin{tabular}{l|l} 
CUADRO 1 & Instrumentos normativos sobre crimen organizado
\end{tabular}

Instrumento normativo

Ley 30077

Artículo 317 Código Penal

Decreto Legislativo núm. 1106

Decreto Legislativo núm. 957

Decreto Legislativo núm. 1244
Ley contra el crimen organizado

Delito de organización criminal

Lucha eficaz contra el lavado de activos y otros delitos relacionados a la minería ilegal y crimen organizado

Nuevo Código Procesal Penal

Decreto legislativo que fortalece la lucha contra el crimen organizado y la tenencia ilegal de armas 
de Justicia de la República, así como la Sala Penal Nacional, han aprobado una serie de acuerdos plenarios vinculantes sobre crimen organizado (ver Cuadro 2). Estos instrumentos tienen como objetivo poner fin a la disparidad de criterios interpretativos a nivel jurisprudencial. En ese sentido, se identifican acuerdos plenarios orientados a definir los elementos criminológicos indispensables para la configuración de la organización criminal (AP 01-2017-SPN), así como para la de delitos concretos vin-

\begin{tabular}{l|l} 
CUADRO 2 & Acuerdos plenarios vinculantes sobre crimen organizado
\end{tabular}

\section{Acuerdo plenario}

Acuerdo Plenario núm. 8-2007/CJ-116

Acuerdo Plenario núm. 3-2005/ CJ-116

Acuerdo Plenario núm. 3-2008/CJ-116

Acuerdo Plenario núm. 3-2010/ CJ-116

Acuerdo Plenario núm. 7-2011/ CJ-116

Acuerdo Plenario núm. 3-2011/ CJ-116

Acuerdo Plenario núm. 2-2012/ CJ-116

Acuerdo Plenario núm. 1-2008/CJ-116

Acuerdo Plenario núm. 2-2010/ CJ-116

Acuerdo Plenario núm. 4-2009/CJ-116

Acuerdo Plenario núm. 7-2009/CJ-116

Acuerdo Plenario núm. 8-2011/ CJ-116

Acuerdo Plenario núm. o12017-SPN

Acuerdo Plenario núm. O22017-SPN

\section{Descripción}

Diferencias entre las agravantes que en el delito de robo aluden a la pluralidad de agentes y a la actuación delictiva como integrantes de una organización criminal.

Intervención de tres o más agentes, alcances del Artículo $297.6 \mathrm{del}$ Código Penal.

Correo de droga, delito de tráfico ilícito de drogas y la circunstancia agravante del artículo 297.6 del Código Penal.

Delito de lavado de activos.

Delito de lavado de activos y medidas de coerción reales.

Delitos contra la libertad sexual y trata de personas: diferencias y penalidad.

Diferencias entre delitos de extorsión o receptación en caso de vehículo automotor objeto de hurto o robo.

Sobre reincidencia y habitualidad.

Concurrencia de circunstancias agravantes específicas de distinto grado o nivel y determinación judicial de la pena.

Determinación de la pena y concurso real de delitos.

Personas jurídicas y consecuencias accesorias.

Beneficios penitenciarios, terrorismo y criminalidad organizada.

Enfoque criminológico y jurídico de la estructura en la organización criminal.

Utilización de la declaración del colaborador eficaz. 
culados al crimen organizado, tales como el lavado de activos (AP o3-2010/CJ-116) o el tráfico ilícito de drogas (03-2008/CJ-116). Asimismo, se encuentran diversos acuerdos vinculantes que desarrollan los criterios para la aplicación de las normas procesales para casos de crimen organizado o de técnicas especiales de investigación, como la figura de colaboración eficaz (AP 02-1017-SPN).

Finalmente, y de manera más reciente, el Estado peruano ha considerado necesario complementar la normativa penal y procesal penal contra el crimen organizado con instrumentos de política dirigidos a combatir de manera más eficaz la presencia del crimen organizado en el Perú. Como se aprecia en el Cuadro 3, desde el año 2013 se ha incrementado el número de políticas y estrategias nacionales encaminadas a fortalecer el control de las diferentes manifestaciones del crimen organizado a nivel de delitos específicos.

CUADRO 3 Políticas nacionales vinculadas al control del crimen organizado

Política o estrategia nacional |

Descripción

Política nacional de seguridad ciudadana 2013-2018 (DS. núm. o12-2013-IN)

Política nacional frente a los delitos patrimoniales (DS. núm. oo6-2014JUS)

Política nacional frente a la trata de personas y sus formas de explotación (DS. núm. oo1-2015JUS)

Política nacional penitenciaria 20162020 (DS núm. 05-2016-JUS)
El objetivo general de la política nacional de seguridad ciudadana es disponer de un Sistema Nacional de Seguridad Ciudadana articulado, para ello, detalla cinco objetivos estratégicos orientados a la implementación de medidas de prevención de la delincuencia, así como de fortalecimiento de las diferentes agencias del sistema de justicia penal involucradas en el control de la criminalidad.

La política nacional frente a los delitos patrimoniales se estructura a partir de tres ejes estratégicos relacionados con los factores de riesgo, las oportunidades de comisión y el acceso a medios para la comisión de delitos patrimoniales. Asimismo, cuenta con diez lineamientos generales y 35 específicos, orientados a conseguir los ejes estratégicos planteados.

Esta política propone prevenir, controlar y reducir la trata de personas a nivel nacional. Para ello, establece tres ejes de intervención asociados a la prevención, persecución del delito y protección a la víctima; y un eje trasversal de focalización y articulación local de las estrategias implementadas para la prevención y control del fenómeno.

La política nacional penitenciaria 2016-2020 se estructura a partir de tres ejes que vinculan a los principales órganos del Sistema de justicia penal, tratamiento y resocialización involucrados en la administración penitenciaria. Cuenta con seis lineamientos generales y 23 específicos, orientados a modernizar el sistema penitenciario y a dotarlo de mayores recursos para su correcto funcionamiento. 
Política nacional contra el lavado de activos y el financiamiento del terrorismo (DS. núm. 018-2017-JUS)
El objetivo general de esta política es mejorar las capacidades del Estado peruano para prevenir, detectar, investigar y sancionar los casos de lavado de activos y financiamiento del terrorismo de manera eficaz y eficiente. Para ello, establece tres ejes estratégicos relacionados con el objetivo general y consigna como eje trasversal la articulación de las estrategias propuestas. Asimismo, concreta cuatro objetivos estratégicos, 16 lineamientos y 18 estándares nacionales de cumplimiento.
Política nacional de integridad y lucha contra la corrupción (DS núm. 092-2017-PCM)
Esta política se estructura a partir de tres ejes que tienen en común fortalecer la capacidad del Estado peruano para prevenir y sancionar los actos de corrupción. En ese sentido, el objetivo general está orientado a fortalecer la transparencia e integridad de los distintos actores de la sociedad: instituciones públicas, sociedad civil, sector privado; así como reforzar las capacidades de cada uno de estos actores en las estrategias anticorrupción.
Estrategia nacional de lucha contra las drogas 2017-2021 (DS núm. o612017-PCM)
Esta estrategia nacional tiene como objetivo general proteger a la sociedad de los efectos negativos, a todo nivel, del comercio ilícito de drogas y de su consumo. Para ello, plantea como objetivo prioritario fortalecer la presencia del Estado peruano en las zonas de influencia cocalera a nivel nacional.
Estrategia de lucha contra la minería ilegal en áreas naturales protegidas de administración nacional 2017-2021 (RP núm. 249-2017-SERNANP)
Esta estrategia propone como objetivo primordial reducir y erradicar la presencia de minería ilegal en las áreas naturales protegidas a nivel nacional y en aquellos lugares que por su ubicación puedan interferir con la creación y conservación de áreas naturales protegidas (zonas de amortiguamiento). Para ello, establece cuatro acciones estratégicas asociadas a la interdicción de la minería ilegal, a la implementación de medidas preventivas, de control de daños y de recuperación de las áreas naturales protegidas afectadas por la minería ilegal y la propuesta de actividades económicas alternativas.
Estrategia nacional para reducir el tráfico ilegal de fauna silvestre en el Perú 2017-2027 (DS. O11-2017-MINAGRI)
El objetivo general de esta estrategia es reducir el tráfico ilegal de la fauna silvestre a nivel nacional. Para ello, se plantea tres objetivos específicos que buscan sensibilizar a la sociedad civil sobre los efectos negativos del tráfico ilegal de especies silvestres, fortalecer la capacidad de control y establecer alianzas binacionales para detectar los casos de tráfico ilícito de fauna silvestre.
Esta política nacional tiene cuatro objetivos específicos orientados a fortalecer las diferentes capacidades del Estado peruano, para 1) controlar y perseguir a las organizaciones criminales, 2) controlar la presencia de mercados ilegales a nivel nacional; 3 ) prevenir la vinculación de la población con el crimen organizado, y 4) fortalecer las acciones de asistencia y auxilio a las víctimas del crimen organizado. 
Del análisis de este panorama se identifica que, debido a la amplia diversidad normativa, la coexistencia de normas previstas en el Código Penal y la de leyes o decretos legislativos complementarios, hay dificultad en su aplicación. Además, existe una deficiente técnica legislativa, agravada por las continuas modificaciones de las que han sido objeto algunas de las normas reseñadas.

En segundo lugar, se advierte cierta dispersión y falta de uniformidad en el diseño y formulación de los instrumentos y estrategias. A nivel metodológico, se han identificado: 1) falta de estructuras claras en los documentos, ya que mientras algunos enuncian de forma directa sus objetivos otros solo mencionan de forma general lineamientos o ejes, sin precisar metas o indicadores; 2 ) falta de asesoramiento técnico especializado, a pesar de la reciente asistencia técnica de organizaciones internacionales y de la publicación de la "Guía de políticas nacionales del centro nacional de planeamiento estratégico" (Ceplan, 2018); 3) falta de pertinencia de los indicadores orientados a evaluar la implementación de las políticas; y 4) desconexión entre la forma en la cual se plantean las políticas, estrategias y acciones, y las capacidades del Estado peruano para su efectiva implementación.

Ello implica que no existe en el Estado peruano un enfoque unificado de combate, contra el crimen organizado, teniendo en consideración que incluso la última política nacional adoptada no consigue superar del todo estas dificultades. Si bien las políticas por delitos específicos son necesarias para atacar las características de cada fenómeno, la ausencia de un enfoque común sobre lo que constituye crimen organizado, así como la falta de diferenciación entre el enfoque de actividades y de estructura en cada política, hace que resulte difícil hablar de la existencia de una política, e incluso de una estrategia unificada de lucha contra el crimen organizado en el Perú.

Finalmente, a nivel conceptual se advierte una mejora en cuanto a la elaboración de los diagnósticos que definen el fenómeno delictivo planteado. Ello se evidencia en la consulta de fuentes especializadas y la utilización de datos empíricos de las manifestaciones delictivas abordadas. Desafortunadamente, a pesar de dichos avances, muchos de los planteamientos teóricos utilizados no se adaptan a la realidad nacional, lo que impide una comprensión certera del control del fenómeno a nivel nacional. Así, ello genera limitada o nula viabilidad de la implementación de las medidas planteadas o, en ciertos casos, contradice el tenor de las recomendaciones de la literatura especializada. A pesar de ello, han existido algunas estrategias específicas que, si bien no llegaron a formalizarse a nivel de documentos de política institucional y no han logrado tener continuidad, dan cuenta de algún avance en la comprensión y tratamiento de este fenómeno desde los sectores involucrados. 


\section{Hacia una política comprehensiva contra el crimen organizado: estrategia basada en un enfoque desde la organización}

El trabajo de las agencias peruanas de seguridad y justicia contra el crimen organizado ha sido constante; sin embargo, la visión estratégica de focalización de los recursos y operaciones desde los sectores involucrados no siempre ha estado presente, en parte debido a la fragmentación de los instrumentos normativos e institucionales en la materia. No obstante, durante el período 2016-2018, la gestión del Gobierno en materia de seguridad se orientó de manera temporal a diseñar y ejecutar una estrategia comprehensiva de lucha contra el crimen organizado. Así, desde el Ministerio del Interior (Mininter, 2017) se implementó la estrategia denominada "Megaoperativos contra el crimen organizado".

Dicha estrategia recogió experiencias ya existentes en las agencias de seguridad y justicia, enfatizando el trabajo interinstitucional basado en inteligencia, y las orientó a la implementación de acciones sistemáticas dirigidas al combate del crimen organizado, que se había convertido en prioridad nacional. Así, en primer lugar, esta estrategia adoptó un enfoque de crimen organizado desde las actividades, tomando en cuenta las diferencias entre los principales tipos de actividad de las organizaciones criminales con incidencia nacional: el crimen predatorio y las economías ilegales. Con ello, se focalizó el trabajo a partir de la recopilación de indicadores específicos de ambos tipos de actividades por cada región. Asimismo, se adoptó un enfoque desde la estructura, al tener como objetivo investigar y desarticular estructuras criminales complejas y llevar a los involucrados ante la justicia bajo el marco normativo de crimen organizado.

La clave fundamental de esta estrategia fue, en primer lugar, el rol de la inteligencia. Esta, tanto humana como tecnológica, era indispensable para el desarrollo exitoso de estos operativos, ya que la desarticulación de organizaciones criminales es producto de meses de investigaciones lideradas por equipos de inteligencia de la Policía Nacional y el Ministerio Público. Dichos equipos implementaban técnicas especiales de investigación contempladas en el marco de la Ley contra el Crimen Organizado, como el agente encubierto o intervención de las comunicaciones, entre otras. El uso de estas técnicas tenía como finalidad servir como sustento para la ejecución de medidas judiciales, como allanamientos y detenciones, y para los procesos judiciales posteriores (Mininter, 2017). Asimismo, el uso de estas técnicas permitía capturas focalizadas y un uso moderado de la fuerza, lo que contrasta con estrategias de patrullaje ostensivo con altos efectos colaterales empleadas en otros países como Brasil.

Además, es importante mencionar que bajo este esquema se realizaban, de manera periódica, acciones de contrainteligencia y control del personal implicado en estas 
investigaciones, a fin de evitar posibles filtraciones de información o actos asociados a la corrupción de funcionarios. Con ello, se buscaba formar equipos de tarea de élite dentro de las fuerzas del orden, no solo en materia de especialización, sino de integridad. Ello implica, por lo tanto, no solo un cambio de estrategia contra el crimen organizado, sino también un inicio de reformas necesarias en el sector seguridad.

La ejecución de esta estrategia da cuenta de algunos elementos clave para una política pertinente contra el crimen organizado: 1) la incorporación de una visión estatal centrada en la estructura de los grupos criminales, que permita la desarticulación de las organizaciones, así como la prevención de su reestructuración;2) la incorporación de un enfoque de las actividades dentro de la política general de crimen organizado, que no implique simplemente una fragmentación por delitos, sino que permita una categorización en función de las características de los tipos de crimen organizado que tienen mayor relevancia en el país; y 3) una focalización territorial con base en indicadores proxy de estos fenómenos. Con ello, además de los pilares de la estrategia mencionados anteriormente, la sostenibilidad de una política nacional comprehensiva contra el crimen organizado podría ser posible. Sin embargo, como en muchos otros países de la región, diferentes factores dificultan la consolidación de un enfoque integral y comprehensivo de combate contra el crimen organizado.

\section{Desafíos para la consolidación de una política de combate al crimen organizado}

Como se ha descrito en los apartados anteriores, el Estado peruano ha recurrido a diferentes estrategias orientadas a controlar y reducir el crimen organizado. A pesar de avances en la materia, tanto a nivel normativo como de políticas públicas, la puesta en marcha de algunas de esas medidas ha evidenciado que aún persisten desafíos para la consolidación de una política comprehensiva de combate al crimen organizado en el Perú. A continuación, reseñaremos tres de los principales desafíos para esta consolidación: 1) la regulación de medidas eficaces contra el crimen organizado, 2) el déficit de capacidades y los problemas para la comprensión de un fenómeno complejo, y 3) la importancia de la confianza y la cohesión social en territorios donde se implementan medidas contra el crimen organizado.

\section{¿Medidas eficaces contra el crimen organizado?}

La persecución, investigación, procesamiento y sanción de casos de crimen organizado tienen un nivel de complejidad mayor que los casos de delitos comunes. Por ello, en la Ley 30 o77 (Ley contra el Crimen Organizado), se autoriza el uso de las 
siguientes técnicas especiales de investigación: interceptación postal, intervención de las comunicaciones, circulación y entrega vigilada de bienes delictivos, agente encubierto, y acciones de seguimiento y vigilancia. La aplicación de dichas técnicas debe cumplir con los requisitos de idoneidad, necesidad y resultar indispensable para llevar a cabo con éxito la investigación en casos de criminalidad organizada (Art. 7), además de respetar los principios de razonabilidad y proporcionalidad en función al caso concreto en el que se aplica.

Paralelamente a la regulación de las técnicas descritas, el Estado peruano ha aprobado diferentes normas que buscan dotar de eficacia la investigación y la persecución de los delitos de criminalidad organizada. Una de ellas fue el Decreto Legislativo $1307^{5}$, que, entre otras cosas, dispuso la ampliación de la duración de la prisión preventiva a 18 meses, prorrogables por 12 meses adicionales a las personas que se encuentren siendo investigadas por delitos asociados al crimen organizado. Esta medida implica que una persona procesada por un delito previsto en la Ley 30077 podría estar recluida en un centro penitenciario durante cuatro años sin tener una sentencia firme que confirme su responsabilidad por el hecho investigado.

Ello evidencia la priorización de la seguridad pública en detrimento de las garantías y derechos constitucionales de los procesados. En ese sentido, se puede advertir una desnaturalización del fin de estas medidas excepcionales presuntamente más eficaces para la lucha contra el crimen organizado. Así, por ejemplo, destaca el abuso de la prisión preventiva. La frecuente aplicación de esta desvirtúa su naturaleza excepcional, reafirmando una tendencia político-criminal predominante en la región latinoamericana que prioriza la inocuización del delincuente, como lo indica el Centro de Estudios de Justicia de las Américas (2011; Corte Interamericana de Derechos Humanos [CIDH], 2013). La "Relatoría sobre los derechos de las personas privadas de libertad" de la Comisión Interamericana de Derechos Humanos ha manifestado su preocupación por la situación descrita, señalando sus efectos negativos en la protección y garantía de los derechos de las personas (CIDH, 2017).

Además, esto se agrava al no contar con un sistema de información eficiente en la materia, lo cual impide tener un panorama completo sobre la gestión procesal de los casos de crimen organizado y medir la eficacia de las medidas empleadas para controlar el fenómeno. Si bien en el año 2015 se aprobó el reglamento del Sistema de 
Control Reforzado de Internos de Criminalidad Organizada ${ }^{6}$ (Siscrico), actualmente no se encuentra implementado por completo.

Desafortunadamente, la realidad peruana demuestra que no es suficiente la previsión legal para una adecuada implementación de las técnicas especiales de investigación o la aplicación de la prisión preventiva. Por el contrario, su regulación no ha venido acompañada de una dotación de recursos logísticos y administrativos suficientes. Ello se traduce en muchos casos en un uso y aplicación distorsionados de estas medidas, lo que ha puesto en tela de juicio el respeto a los derechos humanos y a las garantías constitucionales del debido proceso y de presunción de inocencia (Vervaele, 2009). Sin duda, este constituye uno de los grandes desafíos a abordar de cara a una lucha más eficiente contra el crimen organizado.

\section{El déficit de capacidades y los problemas para la comprensión de un fenó- meno complejo}

A los desafíos normativos deben sumarse los operativos, vinculados principalmente a las capacidades estatales. La implementación de una política de combate contra el crimen organizado precisa de una serie de capacidades por parte de agentes del Estado de, por lo menos, tres tipos (Depetris, 2011; Isuani, 2015): recursos humanos especializados, recursos tecnológicos y alcance territorial. A partir de una revisión de las declaraciones de los principales responsables de las agencias involucradas en la implementación de esta política, así como de entrevistas informales con agentes del sistema de justicia y algunos datos estadísticos disponibles en fuentes oficiales, realizaremos un breve análisis de la situación actual de estas capacidades en el Perú.

En primer lugar, con relación a la cantidad y calidad de los recursos humanos disponibles, podemos señalar que estos son insuficientes. Así, por ejemplo, el coordinador de las Fiscalías Especializadas en Crimen Organizado (FECOR) declaró, a inicios de 2019, que los recursos destinados a la lucha contra el crimen organizado resultan insuficientes:

No tenemos vehículos, por ejemplo, oficinas adecuadas, y obviamente por falta de recursos del Ejecutivo, y no es porque la fiscal de la Nación no quiera, sino que por más que se tengan el deseo y la voluntad, de dónde [lo sacamos], si no hay plata. (Ideeleradio, 2019)

6. El Siscrico es un sistema informático a cargo del Instituto Nacional Penitenciario peruano, que brinda información sobre la situación penal, procesal y penitenciaria de las personas procesadas y condenadas por delitos asociados al crimen organizado, así como de las visitas recibidas. Aprobado por el Decreto Supremo 005-2015-JUS, publicado el 26 de setiembre de 2015, en el diario El Peruano. 
Este panorama se repite en otras instituciones como la Policía Nacional del Perú y el Poder Judicial. Estas restricciones vienen del presupuesto asignado a las instituciones involucradas en la implementación de la política. Por ejemplo, para el año 2019, el Ministerio de Economías y Finanzas autorizó la transferencia de 32005850 soles para el Ministerio Público (Decreto Supremo 150-2019-EF), lo que representa solo un $10 \%$ del total de 379 ooo ooo solicitados por la institución para el año en curso, causando así malestar entre estas instituciones (La República, 2019).

Sin embargo, no es solo la cantidad, sino la calidad de dichos recursos, entendida como el grado de especialización de los oficiales de policía y fiscales para la investigación de delitos complejos y la implementación de técnicas especiales de investigación, que también es limitada. Esto se relaciona con una limitación estructural importante (aunque debatida, y hasta cierto punto modificada) en la Policía Nacional del Perú, ligada a la configuración de líneas de carrera. La fusión de los cuerpos policiales en 1988 llevó a que desapareciera una línea de carrera especializada en investigación criminal (Costa; Neild, 2007) y, a pesar de recientes reformas (como la modificación de los Lineamientos de Formación, Capacitación, Especialización y Perfeccionamiento de la Policía Nacional del Perú, Resolución Ministerial 116-2019IN) que han buscado recrear una especialización en investigación criminal y otra en seguridad pública, la rotación del personal en diferentes funciones para poder ascender en la carrera dificultan que haya una especialización real. Además, suele ser difícil que aquellos que reciben capacitaciones específicas permanezcan suficiente tiempo en su puesto para poner en práctica y perfeccionar lo aprendido, generando así limitaciones en la formación de capacidades no solo individuales, sino también institucionales, como se refleja en los informes de la Defensoría del Pueblo (2009).

Asimismo, tanto policías como fiscales tienen dificultades para poner en operación el concepto de crimen organizado. Por ejemplo, de las audiencias disponibles en el canal estatal Justicia TV y una revisión de los organigramas de grupos criminales producidos por la Policía Nacional del Perú (Mininter, 2017), se puede detectar una tendencia a enfatizar estructuras jerárquicas en las organizaciones criminales (Vizcarra, 2018a). Además, esta caracterización no siempre contiene los elementos para diferenciar al individuo de su papel en la organización, que debe identificarse en función del elemento teleológico de esta. Finalmente, la investigación a gobiernos subnacionales y partidos políticos también ha representado un desafío para la distinción de roles en estructuras ilegales anidadas dentro de otras legales.

El segundo tipo de capacidades necesarias son los recursos tecnológicos, que implican una cobertura de la mayor parte del territorio nacional. Si bien la Policía Nacional requiere de muchos más recursos en general, existen diferencias en la infraestructura de los departamentos especializados, dado que solo algunos de ellos, 
como la Policía antidrogas, reciben apoyo de la cooperación internacional. Asimismo, los recursos de algunos departamentos de investigación criminal especializados son insuficientes, y muchas veces los oficiales deben usar sus propios recursos financieros y logísticos. La implementación de técnicas de investigación especial no es fácil y requiere de más recursos que los actualmente disponibles. Sin embargo, hay recursos como las instalaciones de escucha del "Proyecto Pisco", en la Dirección Nacional de Inteligencia (DINI), que durante varios años estuvieron paralizados y podían usarse para el combate al crimen organizado (Plataforma_GLR, 2015).

En tercer lugar, la capacidad de alcance territorial implica una presencia real eficiente y efectiva en la jurisdicción nacional. Como ya han mostrado investigaciones anteriores (Dargent; Feldmann; Luna, 2017; Rojas, 2016), las autoridades, en algunos casos, no tienen la capacidad suficiente para cubrir todo el territorio nacional, debido tanto al déficit de recursos humanos (lejos del ideal de un policía por cada 250 habitantes) como presupuestal. No obstante, también existen situaciones donde la policía decide no perseguir con el mismo énfasis todos los tipos de delitos (Dargent et al., 2017; Vizcarra, 2018b) en todos los territorios.

\section{Importancia de la recuperación de la confianza y cohesión social en territorios donde se realiza un abordaje operativo del crimen organizado}

Como se ha mencionado en las secciones previas, las estrategias estatales de combate al crimen organizado requieren algunos elementos tanto estratégicos como operativos que, en el caso peruano, empezaron a tener presencia en un período determinado. Algunos de esos elementos tienen que ver con la aproximación a las expresiones del fenómeno a través de algunos indicadores relacionados. Así, el abordaje del fenómeno a este nivel; es decir, desde políticas y estrategias de alcance nacional, sin duda requiere de indicadores pertinentes tanto a nivel regional como local. Sin embargo, incluso teniendo indicadores sólidos a nivel de distritos, algún nivel de aproximación cualitativa al fenómeno es importante.

De esta manera, en el caso del crimen organizado predatorio, por ejemplo, si bien se conoce la evidencia empírica sobre la incidencia delictiva y la presencia de grupos criminales en territorios como las ciudades de la costa norte y centro, no se ha explorado a profundidad cómo es en ellos la relación entre los protagonistas de la actividad criminal y quienes conviven con ella. Algunos estudios de caso en la materia (Bonilla, 2019) señalan que es posible identificar dos momentos en la relación entre este tipo de organizaciones criminales y su entorno barrial más cercano: uno caracterizado por mayores niveles de cohesión social (Sampson, 2012), eficacia colectiva para el control del delito dentro del barrio (Patillo, 1998), grupos crimina- 
les con cierto grado de legitimidad (Patillo, 1998; Venkatesh, 1997) y, con ello, una relación relativamente armónica entre actores legales e ilegales en el barrio; y otro en el que, tras la acción del Estado y la violencia entre las propias organizaciones, estas se fragmentan y reorganizan, teniendo como resultado territorios con bajos niveles de organización social y eficacia colectiva, y altos niveles percibidos de incidencia delictiva o violencia.

Por otro lado, para el caso del crimen organizado asociado al narcotráfico, se tiene también vasta evidencia de la concentración de la producción de drogas ilegales en ciertas zonas del país, especialmente el Vraem (UNODC, 2018). Sin embargo, también se tienen estudios que plantean la existencia de una defensa de dicha actividad (Vizcarra, 2017; Zevallos, 2016) y de resistencias frente a la acción del Estado, haciendo necesario diversificar las estrategias de intervención. En algunos de estos casos, las estrategias de recuperación de confianza deben desarrollarse antes, durante y después de las operaciones relativas a la seguridad.

Con ello, también es importante plantear, en la discusión sobre las políticas en esta materia, el lugar que tienen las consecuencias de la acción del Estado para el orden social en territorios donde el crimen organizado tiene una importante presencia. Es decir, si bien el componente represivo y de control de la política contra el crimen organizado es primordial, también es importante emprender acciones para la recuperación de la confianza en los territorios donde actúa el crimen organizado $\mathrm{y}$, con ello, donde operan las fuerzas del orden contra el fenómeno.

Lo anterior es particularmente importante en casos como los que se han mencionado, donde las organizaciones criminales tienen legitimidad en los territorios en los que conviven, ya sea porque sus estructuras están intrínsecamente ligadas a la organización social del barrio o porque sus actividades se insertan sin dificultad en las actividades económicas que sostienen la economía local. En dichos casos, además, puede tratarse de territorios donde existe una particular desconfianza hacia las instituciones del Estado, debido precisamente a la presencia predominante de organizaciones criminales, por lo que la desarticulación de organizaciones criminales puede conllevar también a la ruptura de ciertos elementos de la organización social importantes para el orden social, de manera que medidas para la recuperación de la confianza en las instituciones y el fomento de la cohesión social son igual de importantes para evitar la reconfiguración de grupos criminales que la acción de las fuerzas del orden. 


\section{Conclusiones}

Este artículo tuvo por objetivo realizar un balance sobre los avances y desafíos de las respuestas del Estado peruano para combatir el crimen organizado durante el siglo XXI, principalmente desde la adopción de la legislación específica orientada a este combate. Se partió de un análisis de la coherencia teórica y empírica de las diferentes normas, políticas y estrategias existentes en el país orientadas al combate contra este fenómeno para comprobar si existían indicios suficientes que permitieran identificar una política general de combate contra el crimen organizado. El análisis revela que la respuesta del Estado peruano frente a este fenómeno es fragmentada y poco coherente, y que incluso la última política general de combate contra el crimen organizado no logra superar los desafíos para crear una política comprehensiva. Si bien no es necesario que la integralidad de las respuestas del Estado contra el crimen organizado sea expresada en un único documento, sí lo es que los diferentes instrumentos guarden coherencia entre ellos, aspecto que no se evidencia a nivel teórico ni empírico.

En el plano teórico, a partir de la distinción de enfoques centrados en la estructura y en las actividades (Paoli, 2014) relacionadas al crimen organizado, constatamos que son pocas las respuestas estatales existentes que incorporan ambas dimensiones del fenómeno (como la estrategia "Megaoperativos"). En algunos casos, las respuestas se centran en las actividades, aunque no necesariamente a partir de una comprensión de su funcionamiento real, como lo demuestra la elección o ausencia de indicadores en determinadas políticas. En varios casos, a pesar de que la calidad y cantidad de información usada para la formulación de instrumentos públicos ha mejorado, esto no se traduce en un diseño coherente. Incluso algunas políticas, como la de delitos patrimoniales o la de trata de personas, carecen de todo tipo de comprensión teórica sobre el fenómeno del crimen organizado, a pesar de estar comprendidos, al menos parcialmente, dentro de esta categoría. Asimismo, estas políticas omiten menciones a las estructuras relacionadas a dicho fenómeno, orientándose, por ejemplo, a indicadores operativos con relación a las víctimas. Este tipo de indicadores también son necesarios, pero no reflejan obligatoriamente un impacto frente al fenómeno criminal.

En el plano empírico, vemos que las políticas y estrategias contra el crimen organizado no terminan de adaptarse a las realidades locales del fenómeno, principalmente con relación a la caracterización de las estructuras de organizaciones criminales que operan en el país. Si bien existen avances significativos en la materia, principalmente a partir de las precisiones aportadas por el Poder Judicial, así como de la formación de capacidades especializadas de investigación criminal en 
la Policía Nacional y el Ministerio Público, aún hay una tendencia por privilegiar el modelo mafia sobre otros modelos de análisis, incluso en aquellas estrategias que muestran una mayor congruencia teórica. En otros casos, los problemas empíricos están relacionados con la ausencia de información o a la omisión de dimensiones relativas al crimen organizado, como sus manifestaciones territoriales y su enraizamiento en contextos sociales específicos. Ello revela que existen desafíos para la academia (peruana e internacional) de producir mayor y mejor información que permita mejorar el conocimiento empírico sobre el fenómeno, pero también hay desafíos para los propios operadores con relación a su comprensión del fenómeno y sus niveles de especialización. Lógicamente, esta no es una responsabilidad individual institucional, y difícilmente será superable si no se prioriza el combate al crimen organizado.

No obstante, la experiencia peruana muestra que existe la posibilidad de desarrollar estrategias de combate contra el crimen organizado con uso intensivo de técnicas de inteligencia que minimicen el uso de la fuerza ostensiva y disminuyan los niveles de exposición al riesgo de poblaciones civiles. Esto no significa que aquellas experiencias más innovadoras carezcan de críticas, como lo evidencia la breve revisión sobre los cuestionamientos a los usos de prisiones preventivas y abusos frente a derechos fundamentales. Sin embargo, sí muestran que, a partir de la experiencia peruana, se podrían desarrollar modelos de intervención eficientes y eficaces para este combate en la región, lo cual implicaría superar los desafíos para la consolidación de una política comprehensiva e interrogarse sobre la coherencia del énfasis punitivo de algunas de estas políticas, lo que debería ser objeto de futuros análisis. Podrían identificarse como unos primeros pasos hacia un enfoque de combate al crimen organizado que sea compatible con el Estado de derecho y la democracia en América Latina.

\section{Referencias}

Andreas, Peter (2011). Illicit Globalization: Myths, Misconceptions, and Historical Lessons. PoliticalScience Quarterly, 126(3), 403-425. https://doi.org/10.1002/j.1538-165X.2011.tboo706.x

Bonilla, Diana (2019). Organización socialy criminalidad organizada en el norte peruano: el caso de Florencia de Mora, Trujillo [Tesis de maestría]. Pontificia Universidad Católica del Perú, Maestría en Sociología, Lima.

Centro de Estudios de Justicia de las Américas (2011). Prisión preventiva y reforma procesal en América Latina (Vol. II). Santiago de Chile: CEJA. 
Centro Nacional de Planeamiento Estratégico (2018). Guía de Políticas Nacionales. Lima: Ceplan. Chin, Ko-Lin (2014). Chinese Organized Crime. En Oxford Handbook of Organized Crime (pp. 219-233), editado por Letizia Paoli. New York: Oxford University Press.

Chu, Yiu Kong (2005). Hong Kong Triads after 1997. Trends in Organized Crime, 8(3), 5-12. https://doi.org/10.1007/s12117-005-1033-9

Comisión Nacional para una Vida sin Drogas (2014). Situación y tendencias del problema de las drogas en el Perú. Lima: Devida/Presidencia del Consejo de Ministros.

Corte Interamericana de Derechos Humanos (2013). Informe sobre uso de la prisión preventiva en las Américas. Recuperado de https://bit.ly/1mkty3M

Corte Interamericana de Derechos Humanos (10 de marzo de 2017). Comunicado 29/17. Relatoría sobre los derechos de personas privadas de libertad realiza visita a Perú. Recuperado de https://bit.ly/2mbEbxo

Costa, Gino; Neild, Rachel (2007). La reforma policial en el Perú. URVIO, 2, 112-126. https:// doi.org/10.17141/urvio.2.2007.1064

Cuzcano, Víctor (2015). Minería ilegal e informal en el Perú: impacto socioeconómico. Cuadernos de CooperAcción, 2. Recuperado de https://bit.ly/2uLcNPO

Dammert, Manuel; Bensús, Víktor; Sarmiento, Katherine; Prieto, Guillermo (2017). Una aproximación a las fronteras desde los mercados ilegales en el Perú. Lima: Flacso/IDRC/PUCP.

Dargent, Eduardo; Feldmann, Andreas; Luna, Juan Pablo (2017). Greater State Capacity, Lesser Stateness: Lessons from the Peruvian Commodity Boom. Politics and Society, 45(1), 3-34. https://doi.org/10.1177/oo32329216683164

Defensoría del Pueblo (2009). Informe Defensorial 142 - Fortalecimiento de la Policía Nacional del Perú: cinco áreas de atención urgente. Lima: Defensoría del Pueblo.

Depetris, Jorge Augusto (2011). Deportes extremos. Construyendo capacidades estatales en la lucha contra el narcotráfico: caso aduana argentina 2005-2010. Documentosy Aportes en Administración Públicay Gestion Estatal, 17, 81-111. https://doi.org/10.14409/da.v1i17.1272

Gambetta, Diego (1993). The Sicilian Mafia: The Business of Private Protection. London: Harvard University Press.

Gereffi, Gary; Humphrey, John; Kaplinsky, Raphael; Sturgeon, Timothy (2001). Globalisation, Value Chains and Development Globalisation, Value Chains and Development. IDS Bulletin, 32(3). Recuperado de https://www.ids.ac.uk/files/dmfile/gereffietal323.pdf

Hagan, Frank (1983). The Organized Crime Continuum: A Further Specification of a New Conceptual Model. Criminal Justice Review, 8(2), 52-57. 
Hill, Peter (2003). The Japanese Mafia: Yakuza, Law and the State. Oxford: Oxford University Press.

Holmes, Leslie (2009). Crime, Organized Crime and Corruption in Post-Communist Europe and the CIS. Communist and Post-Communist Studies, 42, 265-287. https://doi.org/10.1016/j. postcomstud.2009.04.002

Ideeleradio (2019). Chávez Cotrina cuestiona reducción del presupuesto del Ministerio Público. Recuperado de https://ideeleradio.pe/lo-ultimo/chavez-cotrina-cuestiona-reduccion-del-presupuesto-del-ministerio-publico/

Instituto Nacional de Estadística e Informática (2018). Homicidios en el Perú. Contándolos uno a uno 2011-2017. Lima: INEI.

Instituto Nacional Penitenciario (2019). Informe estadístico. Enero 2019. Lima: Ministerio de Justicia y Derechos Humanos/INPE.

Isuani, Francisco (2015). Conferencia. Las capacidades estatales. En Gestión pública: balancey perspectivas (pp. 25-49), editado por Carlos Alza Barco. Lima: Pontificia Universidad Católica del Perú. Recuperado de http://www.escuela.pucp.edu.pe/mwg-internal/de5fs23hu73ds/ progress?id=H7/fUVDLBG

Kenney, Michael (2007). The Architecture of Drug Trafficking: Network Forms of Organisation in the Colombian Cocaine Trade. Global Crime, 8(3), 233-259. https://doi. org/10.1080/17440570701507794

La República (2019). Aumento presupuestal a la Fiscalía aún es insuficiente. Diario La República. Recuperado de https://larepublica.pe/politica/1467660-aumento-presupuestal-fiscalia-insuficiente/

Le, Vy (2012). Organised Crime Typologies: Structure, Activities and Conditions. International Journal of Criminology and Sociology, 1, 121-131.

Ministerio del Interior (2017). Megaoperativos contra el crimen organizado. Lima: Mininter.

Ministerio Público (2018). Mapas del delito 2013-2017. Elaborados con datos de víctimasy denuncias registradas por el Ministerio Público a nivel nacional. Lima: Ministerio Público/Fiscalía de la Nación.

Morselli, Carlo (2009). Inside Criminal Networks. Montreal: Springer.

Naylor, Robin Thomas (2003). Towards a General Theory of Profit-Driven Crimes. The British Journal of Criminology, 43(1), 81-101.

Oficina de Naciones Unidas para la Droga y el Delito (2002). Results of a Pilot Survey of Forty Selected Organized Criminal Groups in Sixteen Countries. Viena: Global Programme Against Transnational Organized Crime. 
Oficina de Naciones Unidas para la Droga y el Delito (2018). Informe de monitoreo de cultivos de coca 2017. Lima: UNODC.

Paoli, Letizia (2003). Mafia Brotherhoods: Organized Crime, Italian Style. New York: Oxford University Press.

Paoli, Letizia (2014). The Oxford Handbook of Organized Crime. New York: Oxford University Press.

Paoli, Letizia (2016). Towards a Theory of Organized Crime: Some Preliminary Reflections. En Illegal Entrepreneurship, Organized Crime and Social Control. Essays in Honor of Professor Dick Hobbs (pp. 3-18), editado por Georgios A. Antonopoulos. New York: Springer.

Pattillo, Mary (1998). Sweet Mothers and Gangbangers: Managing Crime in a Black Middle Class Neighborhood. Social Forces, 76(3), 747-774. https://doi.org/10.2307/3005693

Plataforma_GLR (2015). Equipo de escuchas para combatir el crimen organizado sigue aún en la DINI. Diario La República. Recuperado de https://larepublica.pe/politica/519667-equipo-de-escuchas-para-combatir-el-crimen-organizado-sigue-aun-en-la-dini/

Policía Nacional del Perú (2019). Anuario policial 2018. Lima: Policía Nacional del Perú.

Prado-Saldarriaga, Victor (2019). Lavado de activos y organizaciones criminales en el Perú. Lima: Idemsa.

Reuter, Peter (1983). Disorganized Crime: The Economics of the Visible Hand. Cambridge: MIT Press.

Rojas, Fátima (2016). Limitaciones para el control de la corrupción en las acciones de interdicción de drogas en el VRAEM. Revista de Ciencia Política y Gobierno, 3(5), 59-83. https:// doi.org/10.1880o/rcpg.201601.003

Sala Penal Nacional (2017). Acuerdo plenario núm. 01-2017. Lima: Poder Judicial.

Sampson, Robert (2012). Great American City: Chicago and the Enduring Neighborhood Effect. Chicago: University of Chicago Press.

Van de Bunt, Henk; Siegel, Dina; Zaitch, Damian (2014). The Social Embeddedness of Organized Crime. En The Oxford Handbook ofOrganized Crime (pp.321-339), editado por Letizia Paoli. Oxford: Oxford University Press.

Varese, Federico (2017). What is Organized Crime? En Organized Crime: Critical Concepts in Criminology (Vol. I, pp. 11-33). London: Routledge.

Venkatesh, Sudhir (1997). The Social Organization of Street Gang Activity in an Urban Guetto. American Journal of Sociology, 103(1), 82-111. https://doi.org/10.1086/231172 
Vervaele, John (2009). Medidas procesales especiales y protección de los derechos humanos. Informe general. Utrecht Law Review, 5(2), 159-201.

Vizcarra, Sofia (2017). Instrumentalización de la imagen de la hoja de coca en las tensiones entre el Gobierno local y el Gobierno nacional en la ciudad de Pichari. Revista de Ciencia Política y Gobierno, 4(7), 133-160. Recuperado de http://revistas.pucp.edu.pe/index.php/ cienciapolitica/article/view/19307

Vizcarra, Sofia (2018a). A caracterização das organizações criminais no Peru: aportes para a compreensão do conceito de crime organizado desde os operadores de justiça. Trabajo presentado en Seminário Internacional GIJAL/ALACIP-UFRGS, Democracia e Justiça na América Latina: para onde vamos?, Porto Alegre.

Vizcarra, Sofia (2018b). La economía moral de la ilegalidad en la ciudad cocalera: significadosy prácticas legitimadoras del narcotráfico en la ciudad de Pichari (200o-2017) [Tesis de doctorado]. Pontificia Universidad Católica del Perú, Departamento de Ciencias Sociales, Doctorado en Sociología, Lima.

Volkov, Vadim (2014). Russian Mafia: Rise and Extinction. En The Oxford Handbook of Organized Crime (pp. 159-176), editado por Letizia Paoli. New York: Oxford University Press.

Von Lampe, Klaus (s.f.). Definitions of Organized Crime. Recuperado de www.organized-crime. de/organizedcrimedefinitions.htm

Von Lampe, Klaus (2016). The Practice of Transnational Organized Crime. En Routledge Handbook of Transnational Organized Crime (pp. 186-200), editado por Felia Allum; Stan Gilmour. Londres: Routledge. https://doi.org/10.4324/9780203698341.ch12

Zevallos, Nicolás (2016). Control y defensa del cultivo de coca en el Perú. Lima: Escuela de Gobierno de la PUCP. 